\title{
Atypical clinical presentation of primary hemophagocytic lymphohistiocytosis with a novel perforin 1 gene mutation
}

\author{
Elena Vrotsos • Manuela Soaita • Ziad Khatib • \\ Carole Brathwaite • A. Filipovich • \\ Morton J. Robinson • Amilcar A. Castellano-Sanchez
}

Received: 5 December 2011 / Accepted: 4 June 2012 / Published online: 22 June 2012

(C) Springer-Verlag 2012

\begin{abstract}
Hemophagocytic lymphohistiocytosis (HLH) is a life-threatening systemic disease characterized by abnormal or impaired function of cytotoxic $\mathrm{T}$ cells and natural killer cells leading to an exaggerated but ineffective immune response. We report a 3-year-old boy who is the only common son of a nonconsanguineous couple from Honduras without any history of hemophagocytic lymphohistiocytosis or other hematologic disorders in any of his half-siblings. He first presented to the emergency department with left leg pain. He underwent a left inguinal hernia repair 4 days prior to his initial presentation. He had several subsequent visits to the ER with abdominal pain, emesis, and headaches and was admitted when his symptoms did not resolve. On admission, he was found to have splenomegaly; elevated C-reactive protein, indirect bilirubin, C3 fraction; and pancytopenia. Bone marrow aspirate revealed paucicellular trilineage hematopoiesis with rare atypical histiocytic cells
\end{abstract}

E. Vrotsos $(\bowtie) \cdot$ M. Soaita $\cdot$ M. J. Robinson •

A. A. Castellano-Sanchez

Department of Pathology and Laboratory Medicine, Mount Sinai

Medical Center,

4300 Alton Rd. Blum Bldg. Rm. 2400,

Miami Beach, FL 33140, USA

e-mail: elena.vrotsos@msmc.com

Z. Khatib $\cdot$ C. Brathwaite $\cdot$ A. A. Castellano-Sanchez Department of Pathology, Miami Children's Hospital, Miami, FL, USA

\author{
A. Filipovich \\ Cincinnati Children's Hospital Medical Center, \\ Cincinnati, OH, USA
}

Z. Khatib - C. Brathwaite - A. A. Castellano-Sanchez

Florida International University,

Miami, FL, USA and no evidence of malignancy. An unequivocal diagnosis of HLH could not be made at that time, but was strongly considered. The patient's neurologic status progressively deteriorated leading to brain death. Postmortem genetic analysis showed the patient to be a compound heterozygote with a $681 \mathrm{C}>\mathrm{T}(\mathrm{R} 232 \mathrm{C}) \mathrm{mu}-$ tation and a novel Perforin 1 missense mutation $659 \mathrm{G}>$ A (G220D).

Keywords Hemophagocytic lymphohistiocytosis . Pancytopenia $\cdot$ Perforin1 gene mutation $\cdot$ Natural killer cells . Missense mutation

\section{Introduction}

Familial hemophagocytic lymphohistiocytosis (FHL), originally described by Farquhar and Claireaux in 1952, as an inherited form of HLH syndrome, is an autosomal recessive immune disorder that most commonly presents with fever, hepatosplenomegaly, and cytopenia. It is characterized by abnormal or impaired function of cytotoxic $\mathrm{T}$ cells and natural killer (NK) cells leading to an exaggerated but ineffective immune response. Multiple triggers of the immune response have been identified including infections, predominantly viral, rheumatic diseases, malignancies, and others. FHL is commonly found in ethnic groups where consanguineous marriages are common [1]. About $70 \%$ of the patients present during the first year of life [2]. The constellation of features such as this patient's age at diagnosis, his atypical clinical presentation, and the novel genetic findings of this case-in the absence of other phenotypic features of the genetic syndromes associated with $\mathrm{HLH}$ - makes our patient unique. 


\section{Case report}

A previously healthy 3-year-old boy, the only common son of a nonconsanguineous couple from Honduras, with no family history of HLH or other hematologic diseases in any of his half-siblings, initially presented to the emergency room with left leg pain. He underwent left inguinal hernia repair 4 days prior to his first visit to the ER. He had several subsequent ER visits with abdominal pain, emesis, dizziness, ataxia, and headaches and was admitted when his symptoms did not resolve. Upon admission, the abdominal exam showed splenomegaly. Analysis of his peripheral blood revealed pancytopenia $[\mathrm{Hb}, 70 \mathrm{~g} / \mathrm{L}$; Hct, $20.1 \%$; white blood cell (WBC), $4.1 \times 10^{9}$; thrombocytes, $\left.119 \times 10^{9} / \mathrm{L}\right]$ with low reticulocytes, neutropenia $\left(0.5 \times 10^{9} / \mathrm{L}\right)$, and slight predominance of lymphocytes. Bone marrow aspiration was paucicellular, with trilineage hematopoiesis, and rare atypical cells with histiocytoid morphology reactive for histiocyte marker HAM56. The rare atypical cells had intracytoplasmic, inconspicuous red cells and nuclear debris. There was no evidence of leukemia/lymphoma, other malignancy, or infection. Bone marrow flow cytometry indicated myeloid hypoplasia. An unequivocal diagnosis of HLH could not be made at the time. MRI of the brain showed extensive, infra- and supratentorial white matter disease which progressed during the hospital course. CSF analysis revealed increased protein level (114 mg/dL) and elevated WBC count, $64 \times 10^{6} \%$ $\mathrm{mm}^{3}$, consisting of monocytes. Skeletal imaging demonstrated geographic, evolving lytic and sclerosing lesions located in his long bones. An extensive laboratory panel excluded bacterial, viral, fungal, or mycoplasmal infections from multiple sites (CSF, blood, and bone). Test results for routine toxicology, metabolic defects, and rheumatic disease were negative. A sickle test revealed a stable sickle cell trait. Additional laboratory tests included: ferritin $(1,449 \mu \mathrm{g} / \mathrm{L})$, LDH $(1,692 \mathrm{U} / \mathrm{L})$, increased C3 fraction (183 $\mathrm{mg} / \mathrm{dL})$, increased C-reactive protein $(17.5 \mathrm{mg} / \mathrm{dL})$, and indirect bilirubin $(1.7 \mathrm{mg} / \mathrm{dL})$. Niether fibrinogen levels nor fasting triglycerides were evaluated in our patient. During his last admission, he developed tonic-clonic seizures which advanced to status epilepticus leading to progressive neurologic deterioration and brain death, 2.5 months from his initial presentation. An autopsy limited to the brain and abdominal organs was performed.

\section{Results}

Gross autopsy findings were organomegaly of the spleen, liver, and kidneys. The brain was edematous, weighed
$1,440 \mathrm{~g}$ (1,141 g expected for his age), and was diffusely softened. Microscopic examination of the spleen, lymph nodes (Fig. 1), vertebral marrow, and brain showed lymphohistiocytosis with hemophagocytosis. Histologically, lymphohistiocytic infiltrates were present in the cerebellum, pons, and brainstem with a perivascular pattern and surrounding parenchymal infiltration. Immunohistochemical stains for CD4 and CD8 showed an overall decrease in the number of CD4+ cells when compared to CD8+ cells, and in addition, a comparison of CD8 and Perforin immunohistochemistry demonstrated lack of perforin (PRF1) staining on the CD8+ cells suggesting a defect in perforin protein expression (Perforin and CD8 controls are shown as insets in Fig. 2). Genomic DNA was isolated from deep frozen brain and liver tissues obtained at autopsy. The entire coding region and exon/intron boundaries of $P R F 1$ were analyzed by PCR and direct sequencing. PRF1 gene is located on chromosome 10q21-22. It consists of 554 amino acids. Two different missense mutations involving the $P R F 1$ gene were demonstrated, $681 \mathrm{C}>\mathrm{T}(\mathrm{R} 232 \mathrm{C})^{*}$ and $659 \mathrm{G}>\mathrm{A}(\mathrm{G} 220 \mathrm{D})$ $* *$, confirming the diagnosis of genetic hemophagocytic lymphohistiocytosis. The latter mutation, $659 \mathrm{G}>\mathrm{A}$ (G220D), is to the best of our knowledge a novel mutation in these patients.

\section{Discussion}

In HLH, there is a defect in the granule-dependent cytotoxic pathway resulting in a hyperinflammatory condition. The CTL/NK cells cannot induce apoptosis of the target cells; however, they continue to be activated and produce signals leading to activation of histiocytes with the consequence of hemophagocytosis, resulting in cytopenia and nonspecific clinical symptoms [2-4]. The revised diagnostic guidelines presented by the Histiocyte Society in 2004 include fever,

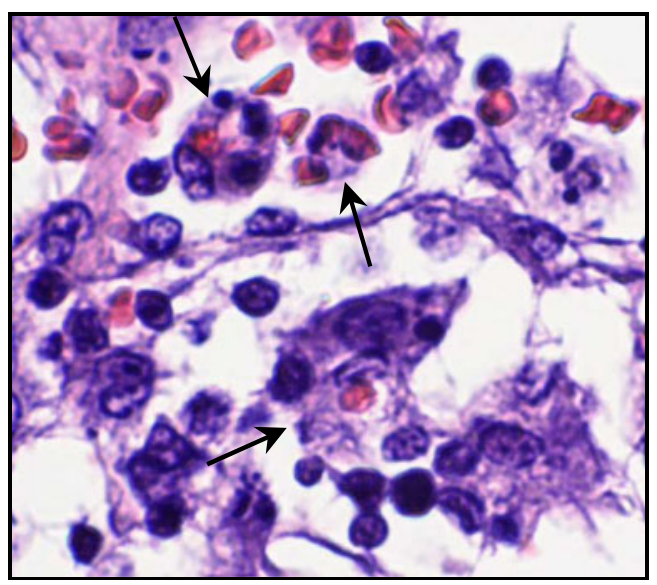

Fig. 1 Prominent histiocytosis with hemophagocytosis in lymph nodes $(\mathrm{HE}, \times 60)$ 
Fig. 2 a Perivascular and parenchymal CD8-positive cells $(\times 40)$, b CD8 expression $\times 200$, c (inset) positive CD8 control, $\mathbf{d}$ absent perforin expression $(\times 40)$, e perforin $(\times 200)$, and $\mathbf{f}$ (inset) positive perforin control

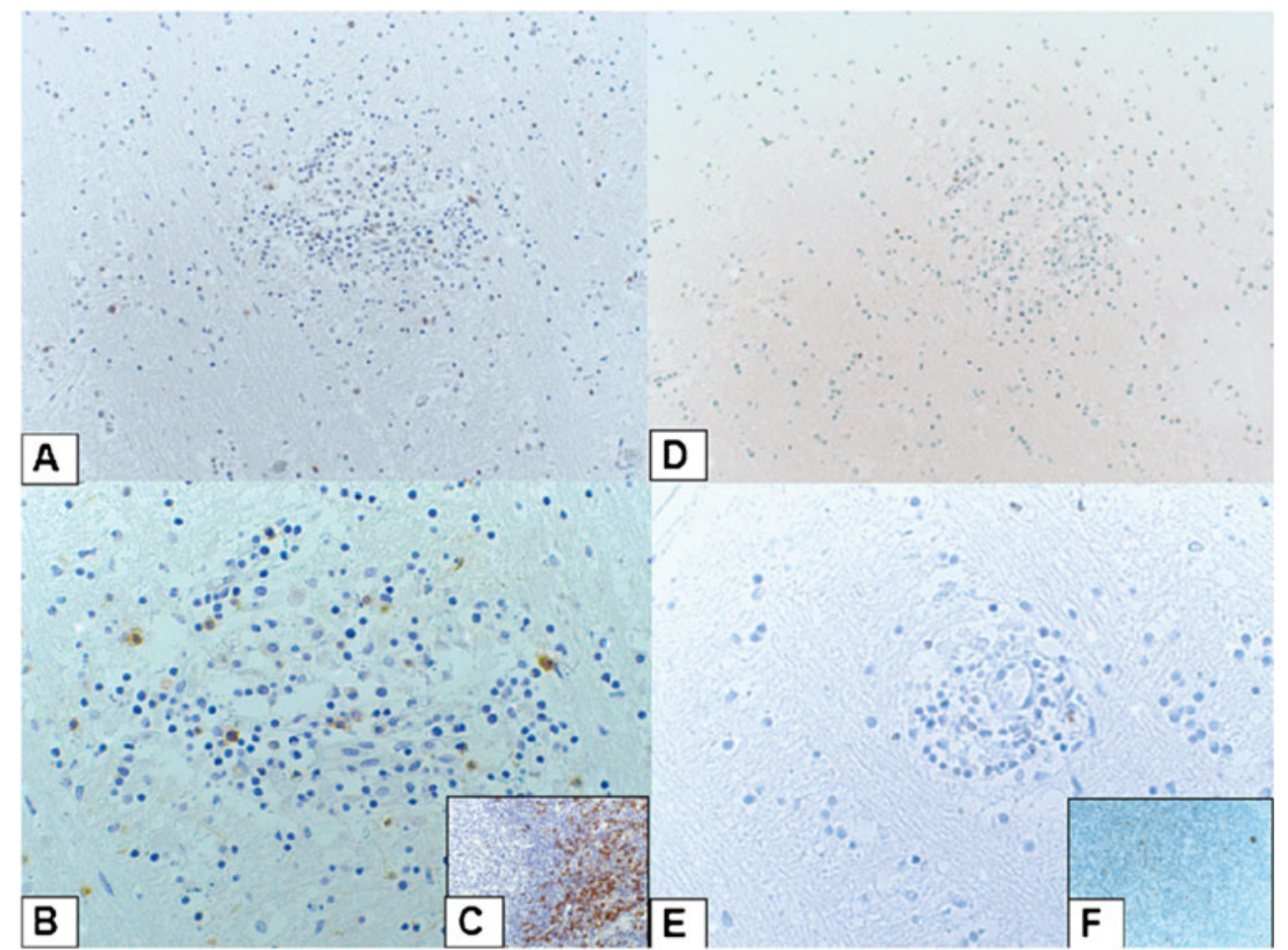

cytopenia, hepatosplenomegaly, common CNS symptoms, biochemical markers of inflammation, and histologic evidence of histiocytic hemophagocytosis [2] (Table 1).

FHL is classified into six different types based on genetic linkage analysis and chromosomal localization. The gene responsible for the type 1 disease is at 9q21.3-22. Type 2 is caused by the mutation in perforin (PRF1) gene at 10q2122. UNC13D (MUNC13-4) gene is located at $17 \mathrm{q} 25$ and is

Table 1 Diagnostic criteria for hemophagocytic lymphohistiocytosis

1. Familial disease/known genetic defect

2. Clinical and laboratory criteria (5/8 criteria)

Fever

Splenomegaly

Cytopenia $\geq 2$ cell lines

Hemoglobin $<90 \mathrm{~g} / \mathrm{L}$ (below 4 weeks $<120 \mathrm{~g} / \mathrm{L}$ )

Neutrophils $<1 \times 10^{9} / \mathrm{L}$

Hypertriglyceridemia and/or hypofibrinogenemia

Fasting triglycerides $\geq 3 \mathrm{mmol} / \mathrm{L}$

Fibrinogen $<1.5 \mathrm{~g} / \mathrm{L}$

Ferritin $\geq 500 \mu \mathrm{g} / \mathrm{L}$

sCD25 $\geq 2,400 \mathrm{U} / \mathrm{mL}$

Decreased or absent NK cell activity

Hemophagocytosis in bone marrow, CSF, or lymph nodes

Supportive evidences are cerebral symptoms with moderate pleocytosis and/or elevated protein in the CSF, elevated blood transaminases and bilirubin, LDH $>1,000$ U/L. Adapted from Janka and Schneider [2] linked to the type 3 (FHL3). Mutations in type 4 occur at $6 \mathrm{q} 24$ with STXII gene, and type 5 has mutations at 19p13.213.3 with $M U N C$ 18-2 gene. Type 6 has no specific mutation.

Since HLH usually is a rapidly fatal condition, early diagnosis is of paramount importance so that appropriate treatment is initiated. There is a recent rapid diagnostic screening approach for patients with FHL-2 in the assessment of PRF-1 gene expression in NK cells by flow cytometry [5]. In addition, a granule release assay analyzing expression of CD107a on the surface of NK and T cells can detect a defect in granule migration, docking, priming, or fusion and is suggestive of FHL-3, FHL-4, and FHL-5 [6].

PRF1 is a cytolytic protein, part of granule-mediated cytotoxicity $[2,7-10]$. It is secreted by cytotoxic CD8+ T lymphocytes, and its role is not only killing the infected or damaged cells but also in halting the immune response [2, 8]. There are multiple recessive mutations in the $P R F 1$ gene. Some of those mutations result in complete or partial reduction in the protein expression or cytotoxic function. Both forms of $\mathrm{HLH}$, genetic or primary (pHLH) and acquired, require a trigger usually infectious, commonly viral $[2,11$, 12], or other various stimuli for $\mathrm{T}$ cell activation (Table 1). Our patient tested negative for bacterial, viral, fungal, and mycoplasmal infections including CMV, EBV, and herpes simplex virus. Of interest, the available literature does not show another case of FLH onset in proximity with a recent surgery. Moreover, the debut at 3 years of age is atypically late as $70 \%$ of the children with pHLH have the onset of the 
disease in the first year of life [2] and only rarely after 2 years of age [13]. The direct causes of death were extensive CNS involvement, massive cerebral edema, and a pontine hemorrhage. The unequivocal histologic evidence of hemophagocytosis in the bone marrow could not be established clinically as the process is typically patchy [2]. Approximately $20-50 \%$ of pHLH are caused by PRF1 mutations, the second most common detected mutation being UNC13D (MUNC13-4) [9, 10, 14]. There have been over 70 reported mutations in the $P R F 1$ gene $[8,15]$. Such $P R F-1$ mutation can result in complete or partial reduction of PRF1 expression, as was shown by IHC in our case (Fig. 2). A review of 34 cases with pHLH showed that six had mutations of the PRF-1 gene, one of them being a heterozygote [9]. Our patient is a compound heterozygote and in addition has a previously unreported pathogenic missense mutation, $659 \mathrm{G}>\mathrm{A}(\mathrm{G} 220 \mathrm{D}) * *$. This missense mutation $659 \mathrm{G}>\mathrm{A}(\mathrm{G} 220 \mathrm{D})^{* *}$ is the disease-causing mutation in our case. It has been previously shown that missense mutation $658 \mathrm{G}>\mathrm{A}$ (G220S) produces the disease resulting in unstable or unfolded protein involving the second transmembrane domain of the gene [16]. The mutation in our case involves the same amino acid; however, the exact mechanism producing the disease has not been established yet. The other mutation $681 \mathrm{C}>\mathrm{T}(\mathrm{R} 232 \mathrm{C})$ is also a missense mutation previously reported to cause the disease [16]. Both mutations involve exon 3 of the $P R F-1$ gene.

There have been many mutations described involving PRF1 gene; however, our patient's presentation including the patient's age, the atypical clinical presentation, and the novel genetic findings make the case unique. It also raises the likelihood of either a nontested viral etiology as the trigger since the infectious workup of the patient was negative, and there are two genetic anomalies known to be capable of initiating the disease; or the possibility of surgical intervention as a contributing factor to disease development.

*Nucleotide sequence alteration, 681 cytosine to thymine; protein sequence alteration, 232 arginine to cysteine.

**Nucleotide sequence alteration, 659 guanine to adenine; protein sequence alteration, 220 glycine to aspartic acid.

Conflict of interest The authors declare that they have no conflict of interest.

\section{References}

1. Gurgay A, Gogus S, Ozyurek E et al (2003) Primary hemophagocytic lymphohistiocytosis in Turkish children. Pediatr Hematol Oncol 20:367-371

2. Janka G, zur Stadt U (2005) Familial and acquired hemophagocytic lymphohistiocytosis. Hematology Am Soc Hematol Educ Program 2005(1):82-88

3. Pradalier A, Teillet F, Molitor J, Drappier J (2004) Revue générale. Syndrome d'activation macrophagique (syndrome d'hémophagocytose). Pathol Biol 52:407-414

4. Wenstrup JR, Zhang K, Filipovich A (March 2006) Cincinnati Children's Hospital Medical Center, Molecular Genetics Laboratory and Immunodeficiency and Histiocytosis Program. Perforin 1 gene mutation analysis report

5. Ménasché G, Feldman J, Fischer A, de Saint Basile G (2005) Primary hemophagocytic syndromes point to a direct link between lymphocyte cytotoxicity and homeostasis. Immunol Rev 203:165-179

6. Ericson K, Fadeel B et al (2001) Spectrum of perforin gene mutations in familial hemophagocytic lymphohistiocytosis. Am J Genet 68:590-597

7. Schneider EM, Lorenz I et al (2002) Hemophagocytic lymphohistiocytosis is associated with deficiencies of cellular cytolysis but normal expression of transcripts relevant to killer-cell-induced apoptosis. Blood 100(8):2891-2898

8. Filipovich A (2006) Cincinnati Children's Hospital Medical Center, Genetic testing for hemophagocytic histiocytosis, Information about genetic testing and genetic counseling, Histiocyte Society

9. Fisman DN (2000) Hemophagocytic syndromes and infection. Synopsis. Emerg Infect Dis 6(6):601-608

10. Allen M, De Fusco, Legrand F et al (2001) Familial hemophagocytic lymphohistiocytosis: how late can the onset be? Haematologica 86:499-503

11. Ueda I, Kohdera U, Hibi S et al (2006) A novel perforin gene mutation in a Japanese family with hemophagocytic lymphohistiocytosis. Int J Hematol 83(1):51-54

12. Ohadi M, Lalloz RA et al (1999) Localization of a gene for familial hemophagocytic histiocytosis at chromosome 9q21.3-22 by homozygosity mapping. Am J Hum Genet 64:165-171

13. Gholam C, Grigoriadou S, Gilmour K, Gaspar H (2011) Familial haemophagocytic lymphohistiocytosis: advances in the genetic basis, diagnosis and management. Clin Exp Immunol 163:271283

14. Murata Y, Yasumi T, Shirakawa R, et al. (2011) Rapid diagnosis of familial hemophagocytic lymphohistiocytosis type 3 (FHL3) by flow cytometric detection of intraplatelet Munc13-4 protein. Blood 2011-01-329540

15. Johnson T, Villanueva J, Filipovich A, Marsh R, Bleesing J (2011) Contemporary diagnostic methods for hemophagocytic lymphohistiocytic disorders. J Immunol Methods 364:1-13

16. Voskoboinik I, Thia M-C, Trapani J (2005) A functional analysis of the putative polymorphisms A91V and N252S and 22 missense perforin mutations associated with familial hemaphogocytic lymphohistiocytosis. Blood 105:4700-4706 